\title{
Arquitectura de redes de información. Principios y conceptos
}

\author{
Architecture of information networks. Principles and concepts
}

\author{
Arquitetura de redes de informação. Princípios e Conceitos
}

\author{
Verónica P. Tintín-Perdomo ${ }^{\mathrm{I}}$ \\ vptintín@espe.edu.ec \\ José R. Caiza-Caizabuano II \\ jrcaiza@espe.edu.ec \\ Fernando S. Caicedo-Altamirano III \\ fscaicedo@espe.edu.ec
}

Recibido: 15 de septiembre de 2017 * Corregido: 20 de noviembre de 2017 * Aceptado: 22 de febrero de 2018

${ }^{\text {I }}$ Ingeniero en Sistemas, Supervisora del Área de Digitalización del Ministerio Coordinador de Desarrollo Social, Docente de la Unidad de Gestión de Tecnologías de la Universidad de las Fuerzas Armadas ESPE, Sangolquí, Ecuador.

II Ingeniero en Sistemas e Informática, Docente de la Unidad de Gestión de Tecnologías de la Universidad de las Fuerzas Armadas ESPE, Sangolquí, Ecuador.

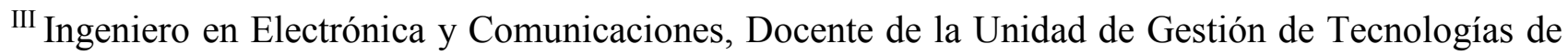
la Universidad de las Fuerzas Armadas ESPE, Sangolquí, Ecuador. 


\title{
Resumen
}

Los grandes avances que se han dado en los últimos años en los campos de tecnologías de la información y las comunicaciones, conducen hacia un mundo cada vez más global e interconectado. La llamada sociedad de la información ha traído consigo un enorme incremento en el número de interacciones entre individuos, así como entre individuos y empresas. La infraestructura que hace posible estas interacciones, son las redes informáticas y su arquitectura. La presente investigación tiene como objetivo estudiar la arquitectura de redes, sus principios y conceptos, con la finalidad de develar que en los entornos de trabajo académico, empresarial, comercial o de cualquier otra índole, el trabajo en red minimiza las redundancias, reduce el tiempo de dedicación y esfuerzo; además de facilitar tanto la difusión como el intercambio de información. La metodología de trabajo fue de tipo bibliográfico, permitiendo una panorámica general de la arquitectura de las redes informáticas, enfocada en los conceptos básicos de los sistemas informáticos, tipos de arquitectura de red informática y tipos de redes. Es de resaltar que en el conocimiento del modelo OSI, en cada una de sus capas, aun cuando tiene dos únicas capas con las cuales interactúa el usuario la primera o capa física y la última o capa de aplicación, existen otras cinco capas donde cada una tiene una función determinada dentro del proceso global de la transmisión de datos entre equipos informáticos interconectados a través de protocolos.

Palabras clave: tecnologías de comunicación e información; arquitectura de redes informáticas; principios; conceptos.

\begin{abstract}
The great advances that have occurred in recent years in the fields of information technology and communications, lead to an increasingly global and interconnected world. The so-called information society has brought with it a huge increase in the number of interactions between individuals, as well as between individuals and companies. The infrastructure that makes these interactions possible is the computer networks and their architecture. The objective of this research is to study the architecture of networks, their principles and concepts, with the purpose of revealing that in academic, business, commercial or any other work environment, networking minimizes redundancies, reduces time of
\end{abstract}


dedication and effort; in addition to facilitating both the dissemination and the exchange of information. The methodology of work was of bibliographic type, allowing a general panoramic of the architecture of the computer networks, focused on the basic concepts of computer systems, types of computer network architecture and types of networks. It is noteworthy that in the knowledge of the OSI model, in each of its layers, even though it has two unique layers with which the user interacts with the first or physical layer and the last layer or application layer, there are five other layers where each one it has a determined function within the global process of the transmission of data between computer equipment interconnected through protocols.

Keywords: communication and information technologies; computer network architecture; beginning; concepts.

\section{Resumo}

Os grandes avanços que ocorreram nos últimos anos nas áreas de tecnologia da informação e comunicações, levam a um mundo cada vez mais global e interconectado. A chamada sociedade da informação trouxe consigo um enorme aumento no número de interações entre indivíduos, assim como entre indivíduos e empresas. A infraestrutura que possibilita essas interações é a rede de computadores e sua arquitetura. O objetivo desta pesquisa é estudar a arquitetura de redes, seus princípios e conceitos, com o objetivo de revelar que, em ambientes acadêmicos, empresariais, comerciais ou qualquer outro ambiente de trabalho, a rede minimiza redundâncias, reduz o tempo de dedicação e esforço; além de facilitar tanto a divulgação quanto o intercâmbio de informações. A metodologia de trabalho foi do tipo bibliográfica, permitindo uma panorâmica geral da arquitetura das redes de computadores, focada nos conceitos básicos de sistemas computacionais, tipos de arquitetura de redes de computadores e tipos de redes. Vale ressaltar que, no conhecimento do modelo OSI, em cada uma de suas camadas, embora tenha duas camadas únicas com as quais o usuário interage com a primeira ou camada física e a última camada ou camada de aplicação, existem outras cinco camadas onde cada uma tem uma função determinada dentro do processo global de transmissão de dados entre equipamentos de informática interconectados através de protocolos.

\section{5} Vol. 4, núm. 2, abril, 2018, pp. 103-122

Verónica P. Tintín Perdomo, José R. Caiza Caizabuano, Fernando S. Caicedo Altamirano 
Palavras chave: tecnologias de comunicação e informação; arquitetura de redes de computadores; princípios; conceitos.

\section{Introducción}

La humanidad, desde épocas prehistóricas, ha evidenciado su gran necesidad de comunicarse, de transmitir información e ideas, con los recursos materiales y tecnológicos con los que ha contado en cada época. Desde las pinturas rupestres y la escritura cuneiforme hasta los modernos y sofisticados sistemas de información y comunicación actuales, la humanidad ha avanzado en su propósito de estar en comunicación con su entorno personal, familiar, laboral, de negocios o simplemente de ocio.

Los grandes avances que se han dado en los últimos años en los campos de tecnologías de información y de comunicaciones (TIC), conducen hacia un mundo cada vez más global e interconectado. La llamada sociedad de la información ha traído consigo un enorme incremento en el número de interacciones entre individuos, así como entre individuos y empresas. La infraestructura que hace posible estas interacciones, son las redes informáticas y su arquitectura (Incera y col, 2007).

Las redes informáticas pueden definirse como un conjunto de equipos (computadoras, periféricos, etc.) que están interconectados y que comparten diversos recursos (Mancilla, 2018). Este tipo de redes implica la interconexión de los equipos a través de ciertos dispositivos que permiten el envío y la recepción de ondas, las cuales llevan los datos que se desea compartir. En las redes informáticas, por lo tanto, hay emisores y receptores que intercambian mensajes.

Por su parte, la arquitectura de red representa la conceptualización o visualización de cómo deben diseñarse y funcionar las redes informáticas en relación con sus propósitos y medios tecnológicos disponibles.

En este trabajo de investigación bibliográfica se presenta una panorámica general de la arquitectura las redes informáticas, se enfoca en los conceptos básicos de sistemas informáticos. 


\section{Desarrollo}

Según Rivera (2016), una red es la interconexión entre dos o más equipos que se comunican entre sí para transmitir o recibir información en distintos instantes de tiempo además de compartir recursos. Dicha alternancia de tiempo se establece a través de mensajes (comando o señales) que determinan un intercambio de roles, bien sea para emitir o para recibir tales mensajes.

Esto, se materializa de un equipo a otro a través de un dispositivo denominado Tarjeta para Interfaz de Red del inglés Network Interface Card (NIC) que maneja un protocolo de comunicación para la emisión/recepción de datos, el cual se denomina nodo de comunicación. A su vez, se comunica a través de un medio de conexión que puede ser cableado o inalámbrico y otro dispositivo que se encarga de conmutar, similar a una central telefónica, las recepciones y emisiones de comunicación llamado switch o conmutador.

Para Peña (2013), los componentes de una red, de manera general los dispositivos conectados a una red informática pueden clasificarse en dos tipos: los que gestionan el acceso y las comunicaciones en una red (dispositivos de red), como módem, router, switch, access point, bridge, entre otros; y los que se conectan para utilizarla (dispositivos de usuario final), como computadora, notebook, tablet, teléfono celular, impresora, televisor inteligente, consola de videojuegos y afines.

En cuanto a la arquitectura de red es el medio más efectivo en cuanto a costos para desarrollar e implementar un conjunto coordinado de productos que se puedan interconectar. La arquitectura es el "plan" con el que se conectan los protocolos y otros programas de software. Esto es benéfico tanto para los usuarios de la red como para los proveedores de hardware y software.

Entre las características de una arquitectura de red, se tiene:

- La separación de funciones. Partiendo de que las redes separan los usuarios y los productos ofertados que evolucionan por la naturaleza de la tecnología con el tipo, las funciones mejoradas se puedan adaptan a la última, a través de la arquitectura de red se concibe el 
sistema de manera modular, para evitar perturbaciones por los cambios mínimos que mantengan una alta disponibilidad.

- Conectividad amplia. Por definición una red debe proveer una conexión óptima entre cualquier cantidad de nodos, considerando los niveles de seguridad requeridos.

- $\quad$ Recursos compartidos. Por medio de las arquitecturas de red puede compartirse recursos tales como impresoras, bases de datos, unidades de disco, haciendo que la operatividad de la red sea económica y eficiente.

- Administración de la red. En la arquitectura se establece la permisología correspondiente para que el usuario defina, opere, cambie, proteja y ejecute mantenimientos en dichared.

- $\quad$ Facilidad de uso. Mediante la arquitectura de red el diseñador puede enfocarse en las interfaces primarias de la red, logrando hacerlas amigables al usuario.

- $\quad$ Administración de datos. Se considera este aspecto unido a la necesidad de interconectar los diferentes sistemas de administración de base de datos, de unidades de almacenamiento y otros servicios.

- $\quad$ Interfaces. Se define las interfaces de persona a red, de persona y de programa a programa. Así la arquitectura combina los protocolos apropiados de software para generar una red funcional.

- Aplicaciones. Aquí son separadas las funciones que se requieren para operar la red de acuerdo con las aplicaciones comerciales de la empresa.

\section{Tipos de arquitectura de red informática}

Como parte de la evolución de las redes informáticas han surgido en el mundo científico y empresarial diversos tipos de arquitectura de redes, algunos de los cuales se describen a continuación. 
- $\quad$ Arquitectura SRA. Fue desarrollada por IBM y describe una estructura integral que provee todos los modos de comunicación de datos y con base en la cual se pueden planear e implementar nuevas redes de comunicación de datos. La ASR se construyó en torno a cuatro principios básicos:

- Comprende las funciones distribuidas con base en las cuales muchas responsabilidades de la red se pueden mover de la computadora central a otros componentes de la red como son los concentradores remotos.

- Define trayectorias ante los usuarios finales (programas, dispositivos u operadores) de la red de comunicación de datos en forma separada de los usuarios mismos, lo cual permite hacer extensiones o modificaciones a la configuración de la red sin afectar al usuario final.

- Se utiliza el principio de la independencia de dispositivo, lo cual permite la comunicación de un programa con un dispositivo de entrada/salida sin importar los requerimientos de cualquier dispositivo único. Esto también permite añadir o modificar programas de aplicación y equipo de comunicación sin afectar a otros elementos de la red de comunicación.

- Se utilizan funciones y protocolos tanto lógicos como físicos normalizados para la comunicación de información entre dos puntos cualesquiera, es decir, que se puede tener una arquitectura de propósito general y terminales industriales de muchas variedades y un solo protocolo de red.

La organización lógica de una red ASR, sin importar su configuración física, se divide en dos grandes categorías de componentes: unidades direccionables de red y red de control de trayectoria.

Las unidades de direccionables de red son grupos de componentes de ASR, que proporcionan los servicios mediante los cuales el usuario final puede enviar datos a través de la red y ayudan a los operadores de la red a realizar el control de esta y las funciones de administración.

La red de control de trayectoria provee el control de enrutamiento y flujo; el principal servicio que proporciona la capa de control del enlace de datos dentro de la red de control de trayectoria es la transmisión de datos por enlaces individuales. La red de control de trayectoria tiene dos capas: la capa de control de trayectoria y la capa de control de enlace de datos. El control de enrutamiento y de flujo 
son los principales servicios proporcionados por la capa de control de trayectoria, mientras que la transmisión de datos por enlaces individuales es el principal servicio que proporciona la capa de control de enlace de datos.

Una red de comunicación de datos construida con base en los conceptos ASR consta de lo siguiente: Computadora principal, Procesador de comunicación de entrada (nodo intermedio), Controlador remoto inteligente (nodo intermedio o nodo de frontera), Diversos terminales de propósito general y orientadas a la industria (nodo terminal o nodo de grupo) y posiblemente redes de área local o enlaces de microcomputadora o macrocomputadora.

- $\quad$ Arquitectura de Red Digital (DRA). Esta es una arquitectura de red distribuida de la Digital Equipment Corporation. Se le llama DECnet y consta de cinco capas. Las capas físicas, de control de enlace de datos, de transporte y de servicios de la red corresponden casi exactamente a las cuatro capas inferiores del modelo OSI. La quinta capa, la de aplicación, es una mezcla de las capas de presentación y aplicación del modelo OSI. La DECnet no cuenta con una capa de sesión separada.

La DECnet, al igual que la ASR de IBM, define un marco general tanto para la red de comunicación de datos como para el procesamiento distribuido de datos. El objetivo de la DECnet es permitir la interconexión generalizada de diferentes computadoras principales y redes punto a punto, multipunto o conmutadas de manera tal que los usuarios puedan compartir programas, archivos de datos y dispositivos de terminal remotos.

La DECnet soporta la norma del protocolo internacional X.25 y cuenta con capacidades para conmutación de paquetes. Se ofrece un emulador mediante el cual los sistemas de la Digital Equipment Corporation se pueden interconectar con las macrocomputadoras de IBM y correr en un ambiente ASR. El protocolo de mensaje para comunicación digital de datos (PMCDD) de la DECnet es un protocolo orientado a los bytes cuya estructura es similar a la del protocolo de Comunicación Binaria Síncrona (CBS) de IBM.

- $\quad$ Arcnet. La Red de computación de recursos conectadas o del inglés Attached Resource Computing Network (ARCNET) es un sistema de red banda base, con paso de testigo (token) que 
ofrece topologías flexibles en estrella y bus a un precio bajo. Las velocidades de transmisión son de 2.5 Mbits/seg. ARCNET usa un protocolo de paso de testigo en una topología de red en bus con testigo, pero ARCNET en sí misma no es una norma IEEE. En 1977, Datapoint desarrollo ARCNET y autorizo a otras compañías. En 1981, Standard Microsystems Corporation (SMC) desarrollo el primer controlador LAN en un solo chip basado en el protocolo de paso de testigo de ARCNET. En 1986 se introdujo una nueva tecnología de configuración de chip.

ARCNET tiene un bajo rendimiento, soporta longitudes de cables de hasta 2000 pies cuando se usan concentradores activos. Es adecuada para entornos de oficina que usan aplicaciones basadas en texto y donde los usuarios no acceden frecuentemente al servidor de archivos. Las versiones más nuevas de ARCNET soportan cable de fibra óptica y de par trenzado. Debido a que su esquema de cableado flexible permite de conexión largas y como se pueden tener configuraciones en estrella en la misma red de área local (LAN Local Área Network).

ARCNET es una buena elección cuando la velocidad no es un factor determinante pero el precio sí. Además, el cable es del mismo tipo del que se utiliza para la conexión de terminales IBM 3270 a computadoras centrales de IBM y puede que va este colocado en algunos edificios. ARCNET proporciona una red robusta que no es tan susceptible a fallos como Ethernet de cable coaxial si el cable se suelta o se desconecta. Esto se debe particularmente a su topología y a su baja velocidad de transferencia. Si el cable que une una estación de trabajo a un concentrador se desconecta o corta, solo dicha estación de trabajo se va a abajo, no la red entera.

El protocolo de paso de testigo requiere que cada transacción sea reconocida, de modo no hay cambios virtuales de errores, aunque el rendimiento es mucho más bajo que en otros esquemas de conexión de red. ARCNET Plus, una versión de $20 \mathrm{Mbits} / \mathrm{seg}$ que es compatible con ARCNET a $2.5 \mathrm{Mbits} / \mathrm{seg}$. Ambas versiones pueden estar en la misma LAN. Fundamentalmente, cada nodo advierte de sus capacidades de transmisión a otros nodos, de este modo si un modo rápido necesita comunicarse con uno lento, reduce su velocidad a la más baja durante esa sesión. ARCNET Plus soporta tamaños de paquetes más grandes y ocho veces más estaciones. 
Otra nueva característica en la capacidad de conectar con redes Ethernet, anillo con testigo y Protocolo de Control de Transmisión/Protocolo Internet (TCP/IP, Transmission Control Protocol/Internet Protocol) mediante el uso de puentes (bridges) y encaminadores (routers). Esto es posible porque la versión nueva soporta la norma de control de enlace lógico IEEE 802.2.

El método de acceso a la ARCnet es mediante un protocolo de bus de token que considera a la red como un anillo lógico. El permiso para transmitir un token se tiene que turnar en el anillo lógico, de acuerdo con la dirección de la tarjeta de interfaz de red de la estación de trabajo, la cual debe fijarse entre 1 y 255 mediante un conmutador DIP de 8 posiciones. Cada tarjeta de interfaz de red conoce su propio modo con la dirección de la estación de trabajo a la cual le tiene que pasar la ficha. El moso con la dirección mayor cierra el anillo pasando la ficha al modo con la dirección menor.

- $\quad$ Ethernet. Desarrollado por la compañía XERTOX y adoptado por la DEC (Digital Equipment Corporation), y la Intel, Ethernet fue uno de los primeros estándares de bajo nivel. Actualmente es el estándar más ampliamente usado. Ethernet esta principalmente orientado para automatización de oficinas, procesamiento de datos distribuido, y acceso de terminal que requieran de una conexión económica a un medio de comunicación local transportando tráfico a altas velocidades. Este protocolo está basado sobre una topología bus de cable coaxial, usando CSMA/CD para acceso al medio y transmisión en banda base a 10 MBPS. Además de cable coaxial soporta pares trenzados. También es posible usar Fibra Óptica haciendo uso de los adaptadores correspondientes.

Además de especificar el tipo de datos que pueden incluirse en un paquete y el tipo de cable que se puede usar para enviar esta información, el comité especifica también la máxima longitud de un solo cable (500 metros) y las normas en que podrían usarse repetidores para reforzar la señal en toda la red.

\section{Funciones de la Arquitectura Ethernet}

- Encapsulación de datos. Formación de la trama estableciendo la delimitación correspondiente, direccionamiento del nodo fuente y destino, así como la detección de errores en el canal de transmisión. 
- Manejo de Enlace. Asignación de canal, resolución de contención, manejando colisiones.

- Codificación de los Datos. Generación y extracción del preámbulo para fines de sincronización Codificación y decodificación de bits.

- Acceso al Canal. Transmisión/Recepción de los bits codificados. Sensibilidad de portadora, indicando tráfico sobre el canal y detección de colisiones, indicando contención sobre el canal.

- Formato de Trama. En una red ethernet cada elemento del sistema tiene una dirección única de 48 bits, y la información es transmitida serialmente en grupos de bits denominados tramas. Las tramas incluyen los datos a ser enviados, la dirección de la estación que debe recibirlos y la dirección de la estación que los transmite Cada interface ethernet monitorea el medio de transmisión antes de una transmisión para asegurar que no esté en uso y durante la transmisión para detectar cualquier interferencia. En caso de alguna interferencia durante la transmisión, las tramas son enviadas nuevamente cuando el medio esté disponible. Para recibir los datos, cada estación reconoce su propia dirección y acepta las tramas con esa dirección mientras ignora las demás. El tamaño de trama permitido sin incluir el preámbulo puede ser desde 64 a 1518 octetos. Las tramas fuera de este rango son consideradas invalidas.

\section{Campos que componen la Trama.}

- El preámbulo. Inicia o encabeza la trama con ocho octetos formando un patrón de 1010, que termina en 10101011. Este campo provee sincronización y marca el límite de trama.

- Dirección destino. Sigue al preámbulo o identifica la estación destino que debe recibir la trama, mediante seis octetos que pueden definir una dirección de nivel físico o múltiples direcciones, lo cual es determinado mediante el bit de menos significación del primer byte de este campo. Para una dirección de nivel físico este es puesto en 0 lógico, y la misma es única a través de toda la red ethernet. Una dirección múltiple puede ser dirigida a un grupo de estaciones o a todas las estaciones y tiene el bit de menos significación en 1 lógico. Para direccionar todas 
las estaciones de la red, todos los bits del campo de dirección destino se ponen en 1, lo cual ofrece la combinación FFFFFFFFFFFFH.

- Dirección fuente. Este campo sigue al anterior, compuesto también por seis octetos, que identifican la estación que origina la trama.

- Los campos de dirección son además subdivididos: Los primeros tres octetos son asignados a un fabricante, y los tres octetos siguientes son asignados por el fabricante. La tarjeta de red podría venir defectuosa, pero la dirección del nodo debe permanecer consistente. El chip de memoria ROM que contiene la dirección original puede ser removido de una tarjeta vieja para ser insertado en una nueva tarjeta, o la dirección puede ser puesta en un registro mediante el disco de diagnóstico de la tarjeta de interfaces de red (NIC). Cualquiera que sea el método utilizado se deber ser cuidadoso para evitar alteración alguna en la administración de la red.

- Tipo. Este es un campo de dos octetos que siguen al campo de dirección fuente, y especifican el protocolo de alto nivel utilizado en el campo de datos. Algunos tipos serian $0800 \mathrm{H}$ para TCP/IP, y 0600H para XNS.

- Campo de dato. Contiene los datos de información y es el único que tiene una longitud de bytes variable que puede oscilar de un mínimo de 46 bytes a un máximo de 1500. El contenido de ese campo es completamente arbitrario y es determinado por el protocolo de alto nivel usado.

- Frame Check Secuence Este viene a ser el último campo de la trama, compuesto por 32 bits que son usados por la verificación de errores en la transmisión mediante el método CRC, considerando los campos de dirección tipo y de dato.

- Modelo SNA. El modelo SNA tiene las siguientes características: Permite compartir recursos, reconoce perdida de datos durante la transmisión, usa procedimientos de control de flujo, evade sobrecarga y la congestión, reconoce fallos y hace corrección de errores. Provee interfaces abiertas documentadas. Simplifica la determinación de problemas gracias a los servicios de administración de 
la red. Mantiene una arquitectura abierta. Provee facilidad de interconexión de redes Provee seguridad a través de rutinas de logon y facilidades de encryptamiento Usa Synchronous Data Link Control (SDLC).

\section{Niveles del Modelo SNA.}

- Niveles de Control del Enlace Físico. El enlace físico de control de capas es la capa o nivel más baja en la arquitectura. Este permite el uso de una variedad realística de medios físicos par la interconexión de procedimientos de control. Procedimientos de protocolos típicos para esta capa o nivel son conexiones físicas provistas por líneas de comunicación, módem y la interface EIA RS-232C. Esta capa o nivel no tan solo permite variar tipos de circuitos punto a punto o multipunto, sino que provee los protocolos físicos para establecer, controlar y liberar los circuitos de datas conmutados.

- Nivel de Enlace de Datos. Los medios de comunicación físicos (ej.: Línea telefónica) requieren técnicas específicas para ser usadas con el fin de transmitir dato entre sistemas a pesar de la naturaleza de tendencia de error de las facilidades físicas. Estas técnicas específicas son usadas en los procedimientos de control de enlace de dato. Las características primarias de la capa o nivel de enlace de Data de IBM SNA es que esta usa Control de Enlace de Data Síncrono (Synchronous Data Link Control - SDLC) como el protocolo de línea de comunicación.

- Nivel de Control de Ruta. Este nivel provee rutas virtualmente libres de errores entre los ultimo orígenes y destinos conectados a la red. Sobre todo, el control de la red abarca o agrupa el establecimiento y manejo de estas rutas a través de la red. El control de sendas o rutas (paths) por lo tanto tiende dos funciones primarias: Enrutar mensajes a través de la red desde el origen hacia las localidades de destino. Segmentar grandes mensajes o combinar pequeños mensajes, llamado segmentar en bloques (blocking), con el propósito de un caudal de transferencia más eficiente a través de la red. 
- Nivel de Control de Transmisión. Provee un control básico de los recursos de transmisión de la red. Las funciones que provee son: Numero de verificación de secuencia cuando se recibe un mensaje. Encriptamiento de datos. Administración de la rapidez en que los requerimientos enviados de una unidad lógica son recibidos en otra unidad lógica. Soporte para las funciones de frontera para nodos periféricos

- Nivel de Control de Flujo de Datos El flujo de datos en una sesión LU-LU necesita ser controlado de acuerdo con los protocolos de sesión usados y este nivel provee ese control. Las funciones que provee este nivel son: Asignación de números de secuencia de flujo de datos Correlación de la petición y respuesta Soporte para protocolos encadenados gracias a que hace agrupamiento en cadenas de las unidades relacionadas de petición Soporte y refuerzo de la petición de sesión y protocolos de modo de respuesta Soporte y coordinación de los modos de transmisión y recepción de los protocolos de sesión

- Nivel de Servicio de Presentación. Los programas de transacciones se comunican unos con otros, de acuerdo con lo bien definidos protocolos de conversación, usando verbos de conversación. Este nivel define estos protocolos para comunicaciones de programa a programa de comunicación. También, controla el uso del nivel de verbos de los programas de transacciones. Controla la carga y el inicio de los programas de transacción, Mantiene y soporta los modos de transmisión y recepción de protocolos de conversación, Supervisa el uso de los parámetros de los verbos de los programas de transacción, Refuerza las restricciones de los protocolos de secuencia y Procesa verbos de programas de transacciones.

- Nivel de Servicios de Transacción. Es el nivel en el que los programas de servicios de transacción son implementados. Provee los siguientes servicios de usuario final: Control operativo del imite de sesión LU-LU Arquitectura de Intercambio de Documentos (DIA) para distribución de documentos entre sistemas de información de oficina basados en SNA Servicios Distribuidos SNA (SNADS) para comunicación asincrónica de datos. 
- El modelo OSI. Según Dordoigne (2015) cada uno de los componentes que conforman la arquitectura de la red se deben gestionar de manera eficiente. En respuesta a ello y otros aspectos, surgió el modelo OSI que da cabida a la compleja cadena de eventos, que se generan con el movimiento de datos en una red.

$\mathrm{Su}$ origen se remonta en la década de los setenta donde la Organización Internacional para la Normalización o International Standars Organization (ISO) donde inicio el desarrollo de un modelo conceptual para la conexión en red llamado Modelo de Referencia de Interconexión de Sistemas Abiertos del Inglés Open Systems Interconnection Reference Model. En 1984, dicho modelo paso a ser un estándar internacional para las comunicaciones en red OSI para especificar como se desplazan los datos dentro una red.

Por su parte Rivera (2016) enfatiza que las especificaciones del modelo OSI son un estándar abierto, es decir, que se encuentra disponible para todos los interesados. También, este modelo contempla siete capas independientes siendo cada una responsable de un grupo de servicios para el proceso de transmisión de la información entre equipos informáticos interconectados a través de protocolos de un nodo a otro, donde cada capa tiene determinada función como parte del proceso global de la transmisión de datos. Cabe destacar que las dos únicas capas con las cuales interactúa el usuario son la primera, capa física y la última, capa de aplicación.

Es importante destacar que cada capa se comunica solo con la siguiente capa inferior y superior de manera estandarizada, permitiendo la implementación independiente de los servicios en cada una de ellas. Las capas actúan como si estuviesen comunicando con su capa homologa en el otro equipo. Ello permite que un proveedor pueda especializarse en un servicio de una capa e integrarla fácilmente con los servicios de las otras capas formando la solución que se requiere.

A continuación, se describe cada capa del modelo OSI (Microsoft, 2017):

- Capa Física. Contempla los elementos físicos de la red, donde se definen las especificaciones eléctricas, mecánicas y funcionales para activar, mantener y desactivar la conexión física. Se 
determinan las características físicas del cableado, codificación de señales, conectores, además de las limitaciones de distancia y velocidad.

- Capa de Enlace. Es la interfaz entre la capa física y de red. Se transforman los paquetes de datos en tramas (unidades de datos), colocando la cabecera de enlace y viceversa, que vienen definidas por la arquitectura de red empleada y el protocolo utilizado.

- Capa de Red. Se encarga del tráfico de la red, direccionando los paquetes de datos además de ocuparse de su entrega. Aquí se convierten las direcciones lógicas en direcciones físicas.

- Capa de Transporte. Se encarga de controlar el flujo de datos entre nodos de manera eficiente para su entrega, además de asegurar que los datos entregados están libres de errores, en secuencia y sin pérdidas o duplicación. También el tamaño de los paquetes está determinado por la arquitectura de la red empleada.

- Capa de Sesión. Establece, administra y termina las sesiones de comunicación, por peticiones y respuestas del servicio entre aplicaciones ubicadas en dos o más computadores conectados en red. Aquí se ubican puntos de control en la secuencia de datos, proporcionando cierta tolerancia a fallos dentro de la sesión de comunicación.

- Capa de Presentación. También se le conoce como el traductor del modelo OSI, porque toma los paquetes de la capa de aplicación, para darles formato a los datos, el tipo de codificación, de conversión de datos incluyendo la compresión/descompresión y el cifrado/descifrado.

- Capa de Aplicación. Es la que habilita la interfaz que emplea el usuario en su computadora, por ejemplo, navegar por la red por el protocolo http (hypertext protocol), enviar un correo electrónico por el protocolo smtp (simple mail transfer protocol) o ubicar un archivo para su descarga desde la red por el protocolo ftp (file transfer protocol). 


\section{Tipos de redes}

Entre los distintos tipos de Redes, se encuentran los siguientes, diferenciados lógicamente por el tamaño y la cantidad de terminales que abarcan:

- LAN: del inglés Local Área Network o Red de Área Local, que se trata de redes pequeñas (hogareñas o empresariales) en donde cada equipo está conectado al resto.

- WAN: del inglés Wide Área Network o Red de Área Extensa, en este caso las redes se dan entre países enteros o inclusive pueden alcanzar una extensión continental.

- MAN: del inglés Metropolitan Área Network o Red de Área Metropolitana, en este tipo de redes la extensión es mucho mayor, abarcando una ciudad o una pequeña población determinada.

También, según el medio físico utilizado las redes se catalogan en:

- Redes alámbricas: utilizan cables para transmitir datos.

- Redes inalámbricas: utilizan ondas electromagnéticas para enviar y recibir información

- Redes mixtas: unas áreas están comunicadas por cable y otras comunicadas de forma inalámbrica.

\section{La topología de red}

Se define como el mapa físico o lógico de una red para intercambiar datos. En otras palabras, es la forma en que está diseñada la red, sea en el plano físico o lógico. El concepto de red puede definirse como "conjunto de nodos interconectados". Un nodo es el punto en el que una curva se intercepta a sí misma. Lo que un nodo es concretamente depende del tipo de red en cuestión.

El éxito de una red está definido por su potencia, rendimiento y alta disponibilidad, además de la libertad de conexión desde el punto de vista del plano de control, sin importar los elementos físicos y lógicos, del plano de datos para los mismos fluyan por el canal de comunicación cumpliendo con los estándares de comunicación sin importar el fabricante. 
Para Banks, Krivan y Linthicum (2015) sobre la topología de red, es importante destacar que no existe una mejor y única topología de red para una empresa. Solo cuando se entienden las principales opciones de topologías, se puede identificar cual es la que funcionara mejor según el tráfico de red o se puede obtener ideas para solucionar problemas en una red existente. A continuación, se describen las topologías de red:

- Red en bus: (bus o "conductor común”) o Red lineal (line) se caracteriza por tener un único canal de comunicaciones (denominado bus, troncal o backbone) al cual se conectan los diferentes dispositivos. Su principal problema son un posible fallo en el cable central y la acumulación de tráfico.

- Red en anillo: (ring) o Red circular, cada estación está conectada a la siguiente y la última está conectada a la primera. Además, puede compararse con la Red en cadena margarita, el cual es un esquema de cableado realizado en cadenas sucesivas entrelazadas (Daisy chain).

- Red en estrella: (star), las estaciones están conectadas directamente a un punto central (concentrador) y todas las comunicaciones se han de hacer necesariamente a través de éste, por cuanto no están conectados entre sí.

- Red en malla: (mesh), cada nodo está conectado a todos los otros, haciéndola muy segura ante un fallo, pero de instalaciones complejas.

- Red en árbol: (tree) o Red jerárquica donde los nodos están colocados en forma de árbol. Desde una visión topológica, la conexión en árbol es parecida a una serie de redes en estrella interconectadas salvo en que no tiene un nodo central. En otras palabras, se trata de una combinación de redes en estrella en la que cada concentrador o switch se conecta a un servidor o a un switch principal.

En la actualidad, las redes emplean principalmente las topologías de tres capas, una que comprende un núcleo de switches que se conectan entre sí y con el proveedor (o los proveedores) de redes

\footnotetext{
120 Vol. 4, núm. 2, abril, 2018, pp. 103-122

Verónica P. Tintín Perdomo, José R. Caiza Caizabuano, Fernando S. Caicedo Altamirano
} 
externo(s), otra capa de usuario o de acceso y una capa de agregación entre estos dos que mueve información.

\section{Conclusiones}

- Se deduce que en un entorno de trabajo académico, empresarial, comercial o de cualquier otra índole, el trabajo en red minimiza las redundancias, reduce el tiempo de dedicación y esfuerzo además de facilitar tanto la difusión como el intercambio de información.

- Es de resaltar y profundizar en el conocimiento del modelo OSI, en cada una de sus capas, aun cuando tiene dos únicas capas con las cuales interactúa el usuario que son la primera, capa física y la última, capa de aplicación, existen otras cinco capas donde cada una tiene una función determinada dentro del proceso global de la transmisión de datos entre equipos informáticos interconectados a través de protocolos.

- El éxito del modelo radica en la estandarización de los protocolos para la transmisión de datos que facilitan la interoperabilidad de sistemas heterogéneos de diversos fabricantes tanto de hardware como de software.

\section{Referencias Bibliográficas}

Banks, E., Krivan, P y Linthicum D. (2015). Cómo cumplir con los requisitos de una buena red. Editorial SearchDataCenter.Es. España.

Dordoigne, J. (2015). Redes informáticas, nociones fundamentales. Edición quinta. Editorial Eni. España.

Incera J., Cartas R., y Cairó O. (2007). Redes Digitales: Presente y Futuro. Instituto Tecnológico Autónomo de México. Visible en: http://allman.rhon.itam.mx/ jincera/IntroRedesDigitales.pdf. Recuperado el: 02/08/2017 
Mancilla CM. (2017). REDES DE COMPUTADORAS. Universidad Nacional del Litoral. Visible en: http://www.fca.unl.edu.ar/informaticabasica/Redes.pdf. Recuperado el 21/08/17

Microsoft. (2017). Definición de las siete capas del modelo OSI y explicación de las funciones. Soporte Técnico. Visible en: https://support.microsoft.com/es-es/help/103884/the-osi-model-s-sevenlayers-defined-and-functions-explained. Recuperado el 17/09/2017.

Peña, C. (2013). Redes la guía definitiva. Editorial REDUSERS. Argentina.

Rivera J. (2016). Fundamentos de redes informáticas. Edición Segunda. Editorial IT Academy Campus. España. 